\title{
Peripheral neuropathy is associated with insulin resistance independent of metabolic syndrome
}

\author{
Ling Han ${ }^{1,2+}$, Lijin $\mathrm{Ji}^{1 \dagger}$, Jing Chang ${ }^{3 \dagger}$, Jian Wen², Wenting Zhao ${ }^{1}$, Hongli Shi ${ }^{1}$, Linuo Zhou ${ }^{1}$, Yiming Li ${ }^{1}$,
} Renming $\mathrm{Hu}^{1}$, Ji Hu${ }^{2 *}$ and Bin $\mathrm{Lu}^{1 *}$

\begin{abstract}
Background: To determine the association of insulin resistance, metabolic syndrome (MetS) with peripheral neuropathy (PN).

Methods: This cross-sectional study consisted of 2035 subjects in Shanghai who were classified as with MetS and without MetS. The new International Diabetes Federation (IDF) criterion was used to define MetS. HOMA-IR was applied to evaluate insulin resistance. All subjects underwent complete foot examination. PN was assessed according to the neuropathy symptom and neuropathy disability scores. Binary logistic regression was performed to analyze the contributions of insulin resistance, features of MetS to PN.

Results: (1) The percentage of PN was $4.0 \%$ in our study. Patients with MetS (47.7\%) had a higher percentage of PN ( $5.5 \%$ vs. $2.6 \%$, respectively, $P=0.001$ ). With the components of MetS increased (non-MetS, three, four, five), a linear increase in the proportion of peripheral neuropathy was observed $(2.6 \%, 4.8 \%, 5.6 \%$ and $7.2 \%$; respectively, P for trend $=0.001)$. (2) In patients with PN, the average age of patients was significantly older than the corresponding non-PN patients. Waist circumference, fasting blood glucose, HbA1c, proportion of treatment for diabetes and hypertension were significantly higher in PN group compared with non-PN group in MetS patients. (3) The frequency of dysglycemia was the highest in PN patients both with and without MetS (96.2\% and 82.1\%, P $=0.084$ ). (4) After adjusting for gender and smoking history, the PN was associated with MetS [odds ratio (OR) 2.0; 95\% confidence interval $(\mathrm{Cl}) 1.2,3.2 ; \mathrm{P}=0.006]$, and age $(\mathrm{OR} 1.1 ; 95 \% \mathrm{Cl} 1.1,1.1 ; \mathrm{P}<0.001)$. When HOMA-IR was added to this binary logistic regression, the association of PN with MetS disappeared $(P=0.110)$, but the PN was still associated with HOMA-IR (OR 1.2; 95\% Cl 1.1, 1.4, P < 0.001).
\end{abstract}

Conclusions: In metabolic syndrome, insulin resistance might play an important role in the development of peripheral neuropathy.

Keywords: Peripheral neuropathy, Dysglycemia, Insulin resistance, Metabolic syndrome

\section{Background}

Peripheral neuropathy $(\mathrm{PN})$ is a prevalent disorder of the peripheral nervous system, which may be associated with varying combinations of weakness, autonomic changes, and sensory changes. Recently, we reported the slight increase of prevalence of PN in impaired glucose

\footnotetext{
* Correspondence: doctor_JiHu@126.com; binlu@fudan.edu.cn

${ }^{\dagger}$ Equal contributors

${ }^{2}$ Department of Endocrinology, the Second Affiliated Hospital of Soochow University, Jiangsu 215004, China

'Department of Endocrinology and Metabolism, Huashan Hospital, Fudan University, No, 12 Wulumuqi Middle Road, Jing'an District, Shanghai 200040 , China

Full list of author information is available at the end of the article
}

regulation (IGR) subjects, and the higher prevalence of PN in type 2 diabetic subjects and older people [1,2]. As an independent risk factor, plasma glucose level may be an important target for strategies to prevent or improve PN [1].

Metabolic syndrome (MetS) is a cluster of dangerous heart attack risk factors: glucose intolerance, central obesity, hypertension and dyslipidemia. People with MetS are at an increased risk for cardiovascular disease and for increased mortality [3]. Recently, several studies showed that the components of MetS had obvious impact on the pathogenesis of PN [4-8].

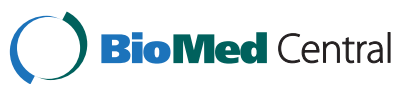

(c) 2015 Han et al.; licensee BioMed Central. This is an Open Access article distributed under the terms of the Creative Commons Attribution License (http://creativecommons.org/licenses/by/4.0), which permits unrestricted use, distribution, and reproduction in any medium, provided the original work is properly credited. The Creative Commons Public Domain Dedication waiver (http://creativecommons.org/publicdomain/zero/1.0/) applies to the data made available in this article, unless otherwise stated. 
Insulin resistance is the core feature of MetS. Data was accumulating to suggest that neurons could also develop insulin resistance, resulting in neuronal injury $[4,9]$. The components of MetS and insulin resistance both might play an important role in the development of PN. However, few data about the influence of insulin resistance, MetS and its individual components on $\mathrm{PN}$ was available in Chinese-based population. Therefore, the aim of this study was to evaluate the association of insulin resistance and MetS with PN in a cross-sectional study.

\section{Research design and methods Study population}

The sample population was recruited from the Shanghai diabetic neuropathy epidemiology and molecular genetics study (SH-DREAMS) from July 2011 to May 2012. SHDREAMS was a population-based cross-sectional study. All non-pregnant community members aged $>25$ years without type 1 diabetes (diagnosed in the past medical history) or renal failure (estimated glomerular filtration rate $<15 \mathrm{ml} / \mathrm{min} / 1.73 \mathrm{~m}^{2}$ or current treatment of kidney dialysis) were invited to participate in our study, and a total of 2149 voluntary individuals were enrolled from 2 communities: Gongkang and Sitang. A total of 114 subjects removed from the study for the following reasons: some difficulties in completing the required tests and the incomplete laboratory measurements. Finally, a total of 2035 subjects, including 728 men and 1307 women, were analyzed. Written informed consents were obtained from all participants. All protocols were approved by Huashan Hospital ethics committee.

\section{Anthropometric measurements}

All participants were asked to complete a questionnaire to collect their demographic information as well as medical history of diabetes and related diseases. Physical examination included measurements of height, weight, waist circumference, and blood pressure. Blood pressure was measured twice using a standard mercury sphygmomanometer and then averaged. Body mass index (BMI) was calculated as weight divided by height squared $\left(\mathrm{kg} / \mathrm{m}^{2}\right)$. Other information such as living habits and lifestyle was also collected.

\section{Laboratory measurements}

After an overnight fast, venous blood samples were collected. Each participant received a 75-g OGTT (oral glucose tolerance test), except for those with a validated history of type 2 diabetes mellitus with $100 \mathrm{~g}$ steamed bread meal test. $100 \mathrm{~g}$ steamed bread meal test, though not a standard test in diabetes research or care, can avoid severe glucose fluctuation in those with a validated history of type 2 diabetes mellitus. Fasting blood samples were analyzed for fasting blood glucose, glycated hemoglobin (HbA1c), fasting insulin and lipid profiles including total cholesterol, triglycerides, high density lipoprotein-cholesterol (HDL-C), and low density lipoprotein-cholesterol (LDL-C). Plasma glucose levels were measured using the glucose oxidase method. Levels of HbA1c were measured by high-pressure liquid chromatography using an analyzer (HLC-723G7, Tosoh Corporation, Japan). Lipid profiles were measured on a Hitachi 7600 analyzer using an enzymatic assay. Homeostasis model assessment of Insulin resistance (HOMA-IR) values were estimated as follows: [fasting blood glucose ( $\mathrm{mmol} / \mathrm{L})$ *fasting insulin $(\mathrm{mU} / \mathrm{L})] / 22.5$. HOMA-IR was applied to evaluate insulin resistance.

\section{Definitions}

MetS was defined by the new International Diabetes Federation (IDF) definition [10,11] if they have three or more of the following: (1) abdominal obesity (waist circumference $\geq 90 \mathrm{~cm}$ for men and $\geq 80 \mathrm{~cm}$ for women); (2) triglycerides $\geq 150 \mathrm{mg} / \mathrm{l}(\geq 1.7 \mathrm{mmol} / \mathrm{l})$ or current use of medication for dyslipidaemia; (3) HDL-C $<1.03 \mathrm{mmol} / \mathrm{l}$ $(<40 \mathrm{mg} / \mathrm{dl})$ for men or $<1.29 \mathrm{mmol} / \mathrm{l} \quad(<50 \mathrm{mg} / \mathrm{dl})$ for women or current use of medication for dyslipidaemia; (4) blood pressure $\geq 130 / 85 \mathrm{~mm} \mathrm{Hg}$ or current use of medication for hypertension; (5) fasting plasma glucose $\geq 5.6 \mathrm{mmol} / \mathrm{l}(100 \mathrm{mg} / \mathrm{dl})$ or current use of medication for diabetes mellitus. Type 2 diabetes and IGR were defined by the ADA definition [1].

\section{PN screening and assessment}

Neuropathy Deficit Score (NDS): Neuropathic deficits in the feet were determined using the NDS, derived from the examination of vibration (using a $128-\mathrm{Hz}$ tuning fork), pin-prick sensation (using Neurotip), temperature sensation (using warm and cool rods), and Achilles tendon reflex (using a tendon hammer). The three perceptions (vibration sense, pain and temperature sensation) were scored 0 if present and normal, and 1 if absent, reduced, or uncertain. On either side, the ankle reflex was scored 0 if present and normal, and 2 if absent [12]. The maximum score was 10 . The severity of neuropathy disability was graded as follows: mild (scores: $3-5$ ), moderate (scores: 6-8), and severe (scores: 9-10).

Neuropathy Symptom Score (NSS): all patients were asked whether they experienced pain or discomfort in their legs. A description of burning, numbness, or tingling was assigned a score of 2 , and fatigue, cramping, or aching was assigned a score of 1 . If the patient described the symptoms as occurring in their feet, calves, and elsewhere, scores of 2, 1 , and 0 were assigned, respectively. Nocturnal exacerbation of symptoms was scored as 2; appearance of symptoms during the day as well as night was scored as 1 , and the symptoms arise during the daytime alone was scored as 0 . If the symptoms had ever woken the patient 
from sleep, a score of 1 was assigned. The patients were asked if any maneuver could reduce their symptoms; walking was assigned a score of 2 , standing 1 , and sitting or lying down 0 . Thus, the maximum symptom score was 9, and the severity of symptoms was graded as follows: mild (scores: 3-4), moderate (scores: 5-6), and severe (scores: 7-9) [13].

The diagnosis of PN depended on both subjective symptoms and signs of neuropathy. We defined $\mathrm{PN}$ as at least moderate signs with or without symptoms (NDS $\geq 6$ ), or mild signs with moderate symptoms (NDS $\geq 3$ and NSS $\geq 5$ ) $[1,12,14]$.

\section{Statistical analysis}

All data was expressed as mean \pm SD or number of cases and percent of individuals affected. A statistical package for SPSS software (version 19.0) was used for statistical analysis. Comparisons between groups were performed using chi-square test or independent $t$ test or Mann-Whitney $\mathrm{U}$ test when appropriate. Binary logistic regression analysis was performed to evaluate the risk factors for PN. Spearman correlation analysis was calculated to assess the relationship among insulin resistance, MetS and PN. The $\mathrm{p}$ value $<0.05$ was considered to be significant.

\section{Results}

A total of 2035 subjects, including 728 men and 1307 women, had an average age of $61.5 \pm 10.1$ years. Among these 2035 subjects, 970 and 1065 subjects were diagnosed with MetS and without MetS. 458, 1043 and 534 subjects were diagnosed with NGT (normal glucose tolerance), IGR and T2DM (type 2 diabetes mellitus) respectively according to the ADA definition after 75-g OGTT tests.

Table 1 presented the clinical characteristics of study population. Among the five groups (NGT, IGR, T2DM, non-MetS and MetS) of subjects, there were no obvious differences between non-PN and PN subjects in the following variables: smoking history, BMI, diastolic blood pressure, HDL-C, and the proportion of patients taking treatment for dyslipidemia. In patients with $\mathrm{PN}$, the average age of patients was significantly older than the corresponding non-PN patients. Duration of diabetes and the proportion of patients taking treatment for dysglycemia were higher in type 2 diabetic patients with $\mathrm{PN}$

Table 1 Clinical characteristics of subjects among NGT, IGR, T2DM, non-MetS and MetS groups

\begin{tabular}{|c|c|c|c|c|c|c|c|c|c|c|}
\hline & \multicolumn{2}{|c|}{$\operatorname{NGT}(n=458)$} & \multicolumn{2}{|c|}{$\operatorname{IGR}(n=1043)$} & \multicolumn{2}{|c|}{ T2DM(n = 534) } & \multicolumn{2}{|c|}{ no-MetS(n = 1065) } & \multicolumn{2}{|c|}{ MetS(n= 970) } \\
\hline & $\begin{array}{l}\text { non-PN } \\
(n=451)\end{array}$ & $\begin{array}{l}P N \\
(n=7)\end{array}$ & $\begin{array}{l}\text { non-PN } \\
(n=1014)\end{array}$ & $\begin{array}{l}P N \\
(n=29)\end{array}$ & $\begin{array}{l}\text { non-PN } \\
(n=489)\end{array}$ & $\begin{array}{l}P N \\
(n=45)\end{array}$ & $\begin{array}{l}\text { non-PN } \\
(n=1037)\end{array}$ & $\begin{array}{l}P N \\
(n=28)\end{array}$ & $\begin{array}{l}\text { non-PN } \\
(n=917)\end{array}$ & $\begin{array}{l}P N \\
(n=53)\end{array}$ \\
\hline Male/All & $141 / 451$ & $0 / 7$ & $348 / 1014$ & $9 / 29$ & $217 / 489$ & $13 / 45^{*}$ & $391 / 1037$ & $9 / 28$ & $315 / 917$ & $13 / 53$ \\
\hline Age(year) & $60.7 \pm 11.0$ & $76.7 \pm 9.7^{*}$ & $60.7 \pm 9.3$ & $71.4 \pm 9.7^{*}$ & $63.5 \pm 9.7$ & $69.6 \pm 9.5^{*}$ & $60.5 \pm 10.2$ & $76.6 \pm 10.6^{*}$ & $61.7 \pm 9.5$ & $70.5 \pm 9.3^{*}$ \\
\hline Smoking history & $68 / 451$ & $0 / 7$ & $134 / 1014$ & $2 / 29$ & $78 / 489$ & $4 / 45$ & $159 / 1037$ & $1 / 28$ & $121 / 917$ & $5 / 53$ \\
\hline $\begin{array}{l}\text { Duration of } \\
\text { T2DM(years) }\end{array}$ & - & - & - & - & $4.2 \pm 6.3$ & $11.2 \pm 9.6^{*}$ & - & - & - & - \\
\hline $\mathrm{BMI}(\mathrm{kg} / \mathrm{m} 2)$ & $23.5 \pm 3.1$ & $25.4 \pm 4.3$ & $24.3 \pm 3.4$ & $24.3 \pm 3.9$ & $25.5 \pm 3.8$ & $25.8 \pm 4.3$ & $23.1 \pm 3.0$ & $24.1 \pm 4.1$ & $25.9 \pm 3.4$ & $25.8 \pm 4.1$ \\
\hline WC(cm) & $82.4 \pm 9.1$ & $90.3 \pm 11.0$ & $85.4 \pm 9.6$ & $88.0 \pm 12.2$ & $90.2 \pm 10.2$ & $92.5 \pm 10.5$ & $81.6 \pm 8.9$ & $85.9 \pm 12.8$ & $90.8 \pm 9.0$ & $93.2 \pm 9.5^{*}$ \\
\hline $\mathrm{SBP}(\mathrm{mmHg})$ & $124.1 \pm 18.4$ & $136.1 \pm 19.5$ & $126.1 \pm 18.6$ & $132.4 \pm 23.6$ & $135.2 \pm 20.9$ & $139.4 \pm 23.0$ & $121.5 \pm 17.8$ & $130.1 \pm 21.6^{*}$ & $135.2 \pm 19.1$ & $140.0 \pm 23.0$ \\
\hline $\mathrm{DBP}(\mathrm{mmHg})$ & $78.7 \pm 10.5$ & $80.6 \pm 10.0$ & $79.8 \pm 10.2$ & $77.4 \pm 12.0$ & $81.7 \pm 10.7$ & $79.7 \pm 11.0$ & $77.1 \pm 9.6$ & $74.6 \pm 10.1$ & $83.3 \pm 10.4$ & $81.3 \pm 11.1$ \\
\hline FBG (mmol/L) & $4.5 \pm 0.6$ & $4.3 \pm 0.3$ & $5.4 \pm 0.8$ & $5.3 \pm 0.8$ & $7.3 \pm 2.7$ & $8.1 \pm 3.8$ & $5.2 \pm 1.5$ & $5.3 \pm 1.4$ & $6.2 \pm 2.0$ & $7.6 \pm 3.6^{*}$ \\
\hline HbA1c (\%) & $5.4 \pm 0.2$ & $5.5 \pm 0.1$ & $5.8 \pm 0.3$ & $5.7 \pm 0.4$ & $7.1 \pm 1.5$ & $7.5 \pm 1.6$ & $5.8 \pm 0.8$ & $6.0 \pm 0.9$ & $6.3 \pm 1.2$ & $7.0 \pm 1.7^{*}$ \\
\hline $\mathrm{TG}(\mathrm{mmol} / \mathrm{L})$ & $1.5 \pm 0.9$ & $1.4 \pm 0.7$ & $1.7 \pm 1.0$ & $1.4 \pm 0.7$ & $2.1 \pm 1.6$ & $1.9 \pm 1.6$ & $1.3 \pm 0.6$ & $1.0 \pm 0.4^{*}$ & $2.2 \pm 1.4$ & $2.0 \pm 1.5$ \\
\hline $\mathrm{TC}(\mathrm{mmol} / \mathrm{L})$ & $5.2 \pm 1.0$ & $5.4 \pm 0.6$ & $5.4 \pm 1.0$ & $5.1 \pm 0.9^{*}$ & $5.5 \pm 1.2$ & $5.5 \pm 1.3$ & $5.3 \pm 1.0$ & $5.3 \pm 1.4$ & $5.5 \pm 1.1$ & $5.3 \pm 1.0$ \\
\hline $\mathrm{HDL}-\mathrm{C}(\mathrm{mmol} / \mathrm{L})$ & $1.4 \pm 0.3$ & $1.3 \pm 0.2$ & $1.4 \pm 0.3$ & $1.3 \pm 0.4$ & $1.3 \pm 0.3$ & $1.2 \pm 0.3$ & $1.5 \pm 0.3$ & $1.5 \pm 0.3$ & $1.2 \pm 0.3$ & $1.2 \pm 0.3$ \\
\hline LDL-C(mmol/L) & $3.1 \pm 0.7$ & $3.5 \pm 0.4$ & $3.1 \pm 0.7$ & $2.9 \pm 0.6$ & $3.2 \pm 0.8$ & $3.3 \pm 0.9$ & $3.1 \pm 0.7$ & $3.2 \pm 1.0$ & $3.2 \pm 0.8$ & $3.2 \pm 0.7$ \\
\hline $\begin{array}{l}\text { Treatment } \\
\text { of diabetes }\end{array}$ & - & - & $0.2 \%$ & $3.4 \%$ & $36.0 \%$ & $71.1 \% *$ & $4.0 \%$ & $17.8 \% *$ & $14.9 \%$ & $52.8 \% *$ \\
\hline $\begin{array}{l}\text { Treatment of } \\
\text { dyslipidemia }\end{array}$ & $2.9 \%$ & $14.3 \%$ & $1.1 \%$ & $0.0 \%$ & $2.2 \%$ & $6.7 \%$ & $0.9 \%$ & $3.6 \%$ & $2.8 \%$ & $5.7 \%$ \\
\hline $\begin{array}{l}\text { Treatment of } \\
\text { hypertension }\end{array}$ & $22.7 \%$ & $28.6 \%$ & $22.8 \%$ & $44.0 \% *$ & $43.6 \%$ & $45.5 \%$ & $16.0 \%$ & $35.3 \% *$ & $41.6 \%$ & $49.0 \%$ \\
\hline
\end{tabular}

Categorical variables were expressed as numbers or percentages.

Continuous variables were expressed as mean \pm SD.

NGT: normal glucose tolerance; IGR: impaired glucose regulation; T2DM: type 2 diabetes mellitus; MetS: metabolic syndrome; PN: peripheral neuropathy; BMI: body mass index; WC: waist circumference; SBP: systolic blood pressure; DBP: diastolic blood pressure; HbA1c: glycated hemoglobin A1c; FBG: fasting blood glucose; HDL-C: high density lipoprotein-cholesterol; LDL-C: low density lipoprotein-cholesterol.

*significantly different from non-PN and corresponding PN groups ( $\mathrm{P}$ value $<0.05$ ). 
than non-PN. Moreover, waist circumference, fasting blood glucose, HbA1c, proportion of treatment for diabetes and hypertension were significantly higher in PN group compared with non-PN group in MetS patients. Additionally, in non-MetS group, patients with PN had a higher level of systolic blood pressure than patients without PN.

The percentage of PN was $4.0 \%$ in our study. Patients with MetS (47.7\%) had a higher prevalence of PN (5.5\% vs. $2.6 \%$, respectively, $\mathrm{P}=0.001)$. $\mathrm{PN}$ was correlated with MetS $(r=0.1, P=0.001)$ and HOMA-IR $(r=0.1, P=0.002)$ by Spearman correlation analysis. A linear increase was observed in the percentage of PN $(2.6 \%, 4.8 \%, 5.6 \%$, and $7.2 \%$, respectively, $\mathrm{P}$ for trend $=0.001$ ) with the increase in the number of MetS components (non-MetS, three, four, five). However, the percentage of PN was not significantly different between men and women $(3.0 \%$ vs. $4.5 \%, \mathrm{P}=0.099)$.

Figure 1 showed the frequencies of MetS components in PN patients. As shown, the frequency of dysglycemia was the highest in PN patients both with and without MetS $(96.2 \%$ and $82.1 \%, P=0.084)$. The higher frequencies of dyslipidemia $(\mathrm{P}<0.001)$ and abdominal obesity $(\mathrm{P}=0.001)$ in MetS group were also observed than nonMetS group in PN patients.

Binary logistic regression was performed to analyze the contributions of insulin resistance and features of the MetS to PN. HOMA-IR was applied to evaluate insulin resistance. After adjusting for gender and smoking history, the $\mathrm{PN}$ was associated with MetS [odds ratio (OR) 2.0; 95\% confidence interval $(\mathrm{CI}) 1.2,3.2 ; \mathrm{P}=0.006]$, and age (OR 1.1; 95\% CI 1.1, 1.1; P<0.001). However, when HOMA-IR was added to this binary logistic regression, the association of $\mathrm{PN}$ with MetS disappeared $(\mathrm{P}=0.110)$, but the PN was associated with HOMA-IR (OR 1.2; 95\% CI 1.1, 1.4, P $<0.001$ ) (Table 2).

\section{Discussion}

Our present study showed the percentage of PN was 4.0\% in a community-based Chinese population. The Italian
General Practitioner Study Group interviewed 4191 elderly subjects (66.5\% were $\geq 65$ years) in northern Italy and found possible neuropathy in $8.0 \%$ [15]. In comparison, only $34.4 \%$ of this Chinese population was $\geq 65$ years. The National Health and Nutrition Examination Survey (NHANES) study revealed a $14.8 \%$ prevalence of PN among 2873 individuals aged $\geq 40$ years and also observed that MetS affected $34.0 \%$ of adults in the US [16,17]. The marked variation in the prevalence of PN might be due to several reasons. Firstly, it was possibly attributed to the wide variation in definitions of PN. In NHANES study, $\mathrm{PN}$ was defined as more than one insensate area according to monofilament testing. While in our study, the diagnosis of PN was based on NDS and NSS. Monofilament testing is a simple and convenient method to diagnose $\mathrm{PN}$, but the sensitivity and specificity in diagnosis of PN remain controversial. Secondly, individuals recruited by previous studies had higher frequency of smoking history than subjects observed in our study $(47.0 \%$ vs. $14.1 \%)$, which contributed to a higher prevalence of PN. Thirdly, race difference might cause a variation in prevalence of PN.

Our results showed that the average age in PN group was significantly older than the corresponding non-PN group, and PN was independently associated with age. These results supported evidences that age was an important risk factor for the development of PN. However, the PN definition was based on NDS and NSS in our study, and NDS might be affected by the age-related reduction of lamellar corpuscles at sensory nerve endings and the decrease of cutaneous innervation [18].

The current study presented approximately $47.7 \%$ of adults met the criteria for MetS. Compared to the nonMetS group, patients with the MetS had a higher prevalence of PN (5.5\% vs. 2.6\%). Consistent with Costa's study [19], a linear increase in the proportion of PN was observed with the increase in the number of MetS components. The results of binary logistic regression in model 1 showed PN was significantly associated with MetS. In MetS patients of

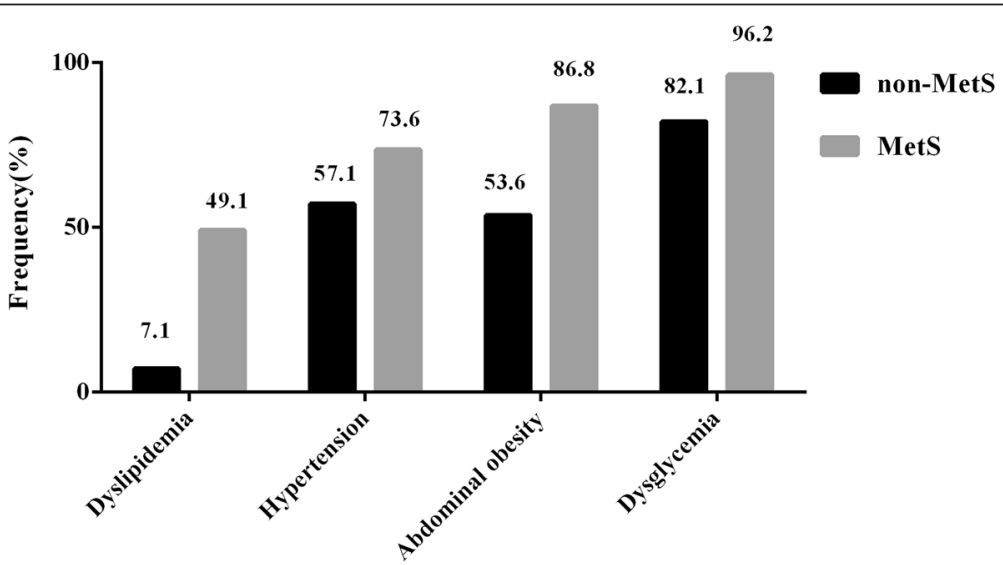

Figure 1 The frequencies of MetS components in PN patients. MetS: metabolic syndrome; PN: peripheral neuropathy. 
Table 2 Binary logistic regression of peripheral neuropathy ${ }^{\mathrm{a}}$

\begin{tabular}{|c|c|c|c|c|c|c|c|c|}
\hline & \multicolumn{4}{|c|}{ Model $1^{b}$} & \multicolumn{4}{|c|}{ Model $2^{c}$} \\
\hline & \multirow[t]{2}{*}{$\mathrm{OR}$} & \multicolumn{2}{|c|}{$95 \% \mathrm{Cl}$} & \multirow[t]{2}{*}{$P$ value } & \multirow[t]{2}{*}{ OR } & \multicolumn{2}{|c|}{$95 \% \mathrm{Cl}$} & \multirow[t]{2}{*}{$P$ value } \\
\hline & & Lower & Upper & & & Lower & Upper & \\
\hline Age & 1.1 & 1.1 & 1.1 & $0.000^{*}$ & 1.1 & 1.1 & 1.1 & $0.000^{*}$ \\
\hline Gender & 1.6 & 0.9 & 2.7 & 0.125 & 1.6 & 0.9 & 2.8 & 0.122 \\
\hline Smoking history & 0.7 & 0.3 & 1.9 & 0.522 & 0.8 & 0.3 & 2.0 & 0.588 \\
\hline MetS-IDF d & 2.0 & 1.2 & 3.2 & $0.006^{*}$ & 1.5 & 0.9 & 2.5 & 0.110 \\
\hline HOMA-IR & I & l & l & I & 1.2 & 1.1 & 1.4 & $0.000^{*}$ \\
\hline
\end{tabular}

a Table 2 showed the binary logistic regression results of peripheral neuropathy. ${ }^{\mathrm{b}} \mathrm{Age}$, gender, smoking history and MetS-IDF were taken into the logistic regression equation in model 1. ${ }^{c}$ And in model 2, HOMA-IR was added to the equation based on model 1. ${ }^{\mathrm{M}}$ MetS-IDF, metabolic syndrome was defined using the new International Diabetes Federation. ${ }^{e *}$ significantly different in the model ( $p$ value $<0.05$ ).

our study, fasting blood glucose and HbA1c were significantly higher in PN group compared with non-PN group. Furthermore, among the PN patients, the frequency of dysglycemia was the highest in both non-MetS and MetS groups. Similar to previous studies, dysglycemia showed the strongest evidence supporting a pathogenic link with PN [20-22]. These results revealed glucose dysregulation, contributing to the diagnosis of MetS, was the leading feature associated with the progression of PN.

Additionally,we also found that, even in non-MS subjects, the higher systolic blood pressure level in PN group was observed. Thus, blood pressure might be also a risk factor for PN. Zarrelli reported hypertension was associated with chronic symmetric polyneuropathy after adjustment for a few common causes of polyneuropathy in Italian subjects aged $\geq 55$ years [23].

Moreover, PN was proved to be independently associated with HOMA-IR after adjusting for MetS in binary logistic regression in model 2. As mentioned above, dysglycemia, as a central feature of MetS, may play a key role in the development of PN. Insulin is the major anabolic hormone regulating the homeostasis of glucose. Insulin resistance is defined as a state of decreased responsiveness of target tissues to normal circulating levels of insulin and is the central feature of type 2 diabetes and MetS [24]. In insulindependent tissues, such as adipose tissue and muscle, the phosphatidylinositol 3-kinase (PI3K)/Akt signaling was crucial for the metabolic effects of insulin, and this pathway was generally affected in individuals with the MetS and diabetes $[4,5]$. Several researches suggested that insulin was one of the growth factors for neurons, and its receptor was widely expressed in nervous systems $[25,26]$. Chronic insulin stimulation was shown to induce insulin resistance in mouse DRGs, as evidenced by decreased activation of Akt and its downstream effectors, and could attenuate the neurotrophic effects of insulin, resulting in mitochondrial dysfunction, subsequent development of PN [27]. These findings indicated that in the MetS insulin resistance might play an important role in the development of PN.
There were several limitations in our study. Firstly, the major limitation of the present study was the criteria used to define neuropathy or in particular nerve damage. The NDS and NSS score is the lack of a rigorous assessment of small fibre damage which is thought to be the earliest nerve fibre deficit and the one likely to occur in IGR. However, an important methodological strength of this study was the use of the OGTT. This enabled us to study the entire spectrum of glucose disorders by identifying subjects with undiagnosed diabetes and pre-diabetes. Secondly, because of serious painful symptoms, patients in PN group had higher percentages of treatment for diabetes and hypertension than subjects without PN. Due to this condition, these data could not reflect the original healthy status of patients. Thirdly, we could not make inferences regarding cause and effect because of the cross-sectional design.

\section{Conclusions}

Insulin resistance might play an important role in the development of peripheral neuropathy in the metabolic syndrome.

\section{Abbreviations}

PN: peripheral neuropathy; IR: Insulin resistance; MetS: Metabolic syndrome; HOMA-IR: Homeostasis model assessment of insulin resistance; FBG: Fasting blood glucose; NGT: Normal glucose tolerance; IGR: Impaired glucose regulation; T2DM: Type 2 diabetes mellitus; OGTT: Oral glucose tolerance test; HbA1c: Glycated hemoglobin A1c; HDL-C: High density lipoprotein-cholesterol; LDL-C: Low density lipoprotein-cholesterol; BMl: Body mass index; WC: Waist circumference; SBP: Systolic blood pressure; DBP: Diastolic blood pressure; IDF: International Diabetes Federation.

\section{Competing interests}

The authors declare that they have no competing interests.

\section{Authors' contributions}

$\mathrm{BL}, \mathrm{RMH}$ conceived and designed the experiments. $\mathrm{LH}$ and $\mathrm{LJ}$ researched data and drafted the manuscript. HLS and LNZ supervised the study design. $\mathrm{BL}, \mathrm{JH}$ wrote, reviewed, and edited the manuscript. WTZ, JC contributed to the statistical analysis. $\mathrm{LH}$ and $\mathrm{JH}$ participated in the discussion. $\mathrm{LH}, \mathrm{LJ}, \mathrm{JC}$, JW, WTZ, YML performed the experiments. All authors read and approved the final manuscript. 


\section{Acknowledgments}

The authors extend appreciation to the participants of the SH-DREAMS. Without their collaboration, this article would not have been written. The authors also thank the SH-DREAMS team involved in patient recruitment and sample and data handling, but who does not meet the criteria for authorship. This study was supported by grants to from the National Natural Science Foundation of China (81370884, to B Lu), the Shanghai New Excellent Youth Program (XYQ2013120, to B Lu), Fudan Zhuoxue Project (to B Lu) and the National Natural Science Foundation of China (81471041, to J Hu), the Science and Technology Development Program of Suzhou(SYS201472, to J Hu).

\section{Author details}

${ }^{1}$ Department of Endocrinology and Metabolism, Huashan Hospital, Fudan University, No, 12 Wulumuqi Middle Road, Jing'an District, Shanghai 200040, China. ${ }^{2}$ Department of Endocrinology, the Second Affiliated Hospital of Soochow University, Jiangsu 215004, China. ${ }^{3}$ Department of the Third Internal Medicine, Affiliated hospital of Shandong Academy of Medical Sciences, No. 38 Wuyingshan Road, Shandong 250031, China.

Received: 21 October 2014 Accepted: 13 February 2015

Published online: 03 March 2015

\section{Reference}

1. Lu B, Hu J, Wen J, Zhang Z, Zhou L, Li Y, et al. Determination of peripheral neuropathy prevalence and associated factors in Chinese subjects with diabetes and pre-diabetes - ShangHai diabetic neuRopathy epidemiology and molecular genetics study (SH-DREAMS). PLoS One. 2013;8:e61053.

2. Lu B, Yang Z, Wang M, Yang Z, Gong W, Yang Y, et al. High prevalence of diabetic neuropathy in population-based patients diagnosed with type 2 diabetes in the Shanghai downtown. Diabetes Res Clin Pract. 2010;88:289-94.

3. Ford ES. Risks for all-cause mortality, cardiovascular disease, and diabetes associated with the metabolic syndrome: a summary of the evidence. Diabetes Care. 2005;28:1769-78.

4. Kim B, Feldman EL. Insulin resistance in the nervous system. Trends Endocrinol Metab TEM. 2012;23:133-41.

5. Visser NA, Vrancken AF, van der Schouw YT, van den Berg LH, Notermans NC. Chronic idiopathic axonal polyneuropathy is associated with the metabolic syndrome. Diabetes Care. 2013;36:817-22.

6. Lee $\mathrm{RH}$, Dellon AL. Insulin resistance. Does it play a role in peripheral neuropathy? Diabetes Care. 1999;22:1914-5.

7. Smith $A G$, Singleton JR. Obesity and hyperlipidemia are risk factors for early diabetic neuropathy. J Diabetes Complicat. 2013;27:436-42.

8. Cameron NE, Eaton SE, Cotter MA, Tesfaye S. Vascular factors and metabolic interactions in the pathogenesis of diabetic neuropathy. Diabetologia. 2001:44:1973-88

9. Callaghan BC, Cheng HT, Stables CL, Smith AL, Feldman EL. Diabetic neuropathy: clinical manifestations and current treatments. Lancet Neurol. 2012;11:521-34.

10. Alberti KG, Eckel RH, Grundy SM, Zimmet PZ, Cleeman II, Donato KA, et al. Harmonizing the metabolic syndrome: a joint interim statement of the international diabetes federation task force on epidemiology and prevention; national heart, lung, and blood institute; American heart association; world heart federation; international atherosclerosis society; and international association for the study of obesity. Circulation. 2009;120:1640-5.

11. Lu B, Zhang S, Wen J, Yang Y, Yang Z, Zhang Z, et al. The New unified international diabetes federation/American heart association/national heart lung, and blood institute metabolic syndrome definition: does it correlate better with C-reactive protein in Chinese patients diagnosed with type 2 diabetes? J Int Med Res. 2010;38:1923-32.

12. Cabezas-Cerrato J. The prevalence of clinical diabetic polyneuropathy in Spain: a study in primary care and hospital clinic groups. Neuropathy Spanish study group of the Spanish diabetes society (SDS). Diabetologia. 1998;41:1263-9.

13. Young MJ, Boulton AJ, MacLeod AF, Williams DR, Sonksen PH. A multicentre study of the prevalence of diabetic peripheral neuropathy in the United Kingdom hospital clinic population. Diabetologia. 1993;36:150-4.

14. Daousi C, MacFarlane IA, Woodward A, Nurmikko TJ, Bundred PE, Benbow SJ. Chronic painful peripheral neuropathy in an urban community: a controlled comparison of people with and without diabetes. Diabetic Med J Bri Diabetic Assoc. 2004;21:976-82.
15. Chronic symmetric symptomatic polyneuropathy in the elderly: a field screening investigation in two Italian regions. I. Prevalence and general characteristics of the sample. Italian General Practitioner Study Group (IGPSG). Neurology. 1995;45:1832-6.

16. Gregg EW, Sorlie P, Paulose-Ram R, Gu Q, Eberhardt MS, Wolz M, et al. Prevalence of lower-extremity disease in the US adult population $>=40$ years of age with and without diabetes: 1999-2000 national health and nutrition examination survey. Diabetes Care. 2004;27:1591-7.

17. Ervin RB. Prevalence of metabolic syndrome among adults 20 years of age and over, by sex, age, race and ethnicity, and body mass index: United States. National Health Statistics Reports. 2006;2009:1-7.

18. Pare M, Albrecht PJ, Noto CJ, Bodkin NL, Pittenger GL, Schreyer DJ, et al. Differential hypertrophy and atrophy among all types of cutaneous innervation in the glabrous skin of the monkey hand during aging and naturally occurring type 2 diabetes. J Comp Neurol. 2007;501:543-67.

19. Costa LA, Canani LH, Lisboa HR, Tres GS, Gross JL. Aggregation of features of the metabolic syndrome is associated with increased prevalence of chronic complications in Type 2 diabetes. Diabetic Med J Bri Diabetic Assoc. 2004;21:252-5.

20. Novella SP, Inzucchi SE, Goldstein JM. The frequency of undiagnosed diabetes and impaired glucose tolerance in patients with idiopathic sensory neuropathy. Muscle Nerve. 2001;24:1229-31.

21. Sumner CJ, Sheth S, Griffin JW, Cornblath DR, Polydefkis M. The spectrum of neuropathy in diabetes and impaired glucose tolerance. Neurology. 2003;60:108-11.

22. Katon JG, Reiber GE, Nelson KM. Peripheral neuropathy defined by monofilament insensitivity and diabetes status: NHANES 1999-2004. Diabetes Care. 2013;36:1604-6.

23. Zarrelli MM, Amoruso L, Beghi E, Apollo F, Di Viesti P, Simone P. Arterial hypertension as a risk factor for chronic symmetric polyneuropathy. J Epidemiol Biostatistics. 2001;6:409-13.

24. Sesti G. Pathophysiology of insulin resistance. Best Pract Res Clin Endocrinol Metab. 2006:20:665-79.

25. Xu QG, Li XQ, Kotecha SA, Cheng C, Sun HS, Zochodne DW. Insulin as an in vivo growth factor. Exp Neurol. 2004;188:43-51.

26. Toth C, Brussee V, Martinez JA, McDonald D, Cunningham FA, Zochodne DW. Rescue and regeneration of injured peripheral nerve axons by intrathecal insulin. Neuroscience. 2006;139:429-49.

27. Dunn TN, Adams SH. Relations between metabolic homeostasis, diet, and peripheral afferent neuron biology. Advances Nutr (Bethesda, Md). 2014:5:386-93.

\section{Submit your next manuscript to BioMed Central and take full advantage of:}

- Convenient online submission

- Thorough peer review

- No space constraints or color figure charges

- Immediate publication on acceptance

- Inclusion in PubMed, CAS, Scopus and Google Scholar

- Research which is freely available for redistribution 